\section{Importance of the Microscopic Wave Function in Envelope-Function Theory}

In two recent Letters [1,2], Krebs et al. discuss experimental data showing strong polarization anisotropy in the optical absorption spectra of zinc-blende semiconductor heterostructures. They interpret the data using a heuristic envelope-function (EF) model that incorporates the reduction in crystal symmetry (from $T_{d}$ to $C_{2 v}$ ) caused by a [001] heterojunction or electric field. This loss of symmetry generates a mixing between heavy and light holes even at zero in-plane wave vector, an effect not included in conventional EF models.

The purpose of this Comment is to point out that, although their model does capture the qualitative features of the broken symmetry, its numerical prediction for the mixing strength is not reliable because it is inconsistent with the microscopic potential energy and wave function. Furthermore, the model is not even self-consistent, since the bulk electric-field mixing (i.e., the Pockels effect) changes sign when one changes the definition of the unit cell. A solution to both problems is presented here.

The correct way to describe interband mixing has been known for many years: It is given by Eq. (II.19) of Luttinger and Kohn's (LK's) paper on effective-mass theory [3]. The LK representation of the wave function is an exact representation valid for any microscopic potential, including that of a heterostructure under an applied electric field. Burt has used this representation to obtain an exact envelope-function theory [4] by transforming LK's Eq. (II.14) directly from $\mathbf{k}$ space to $\mathbf{r}$ space, avoiding the usual "gentle potential" approximation (II.21). The author has shown that Burt's interface Hamiltonian may be interpreted as a simple unit-cell average of the microscopic Hamiltonian and Bloch functions [5]. This unitcell average gives the same interband mixing strength as Eq. (II.19) of Ref. [3].

The calculations of Ref. [5] show that, contrary to the claims in Refs. [1] and [2], the mixing strength at a heterojunction generally cannot be predicted from the band offsets between the materials. It depends strongly on the details of the microscopic interface potential, and potentials with the same band offsets can give rise to coupling strengths differing by more than an order of magnitude [5]. Therefore, in a heuristic theory such as Krebs and Voisin's [1], the mixing strength must always be treated as a fitting parameter (as it is in the symmetrybased theory of Ivchenko et al. [6]).

The electric-field dependence of the mixing in Krebs and Voisin's " $H_{B F}$ " model is determined from the postulate that the first-order correction to standard EF theory is proportional to the first derivative of a slowly varying potential. This sounds reasonable at first, but it contradicts LK's Eq. (II.19), which shows that the coupling is a mixture of the properties of the potential and the Bloch functions, and cannot be written in terms of the potential alone. Inspection of LK's Eqs. (II.14) and (II.19) shows that a slowly varying potential mixes only those parts of the EFs near the Brillouin zone boundary; consequently, the mixing is usually neglected for slowly varying EFs. It clearly does not have the form of the overlap integral given in Eq. (1) of Ref. [1].

In addition to this discrepancy with microscopic theory, the $H_{B F}$ Hamiltonian is found upon closer examination to be internally inconsistent. The potential in this model is defined as [1] $V_{B F}(z)=\sum_{l} h\left(z-z_{l}\right)\left\{\left[V\left(z_{l}\right)-\right.\right.$ $\left.(a / 4) d V / d z] B+\left[V\left(z_{l}\right)+(a / 4) d V / d z\right] F\right\}$, where $V(z)$ is the external potential, $a$ is the lattice constant, $h(z-$ $\left.z_{l}\right)$ is a unit step of width $a / 2$ centered on the $l$ th plane of anions, and $B$ and $F$ are projection operators for $p$ orbitals lying in (110) and (110) planes. As stated in Ref. [1], the choice of anion planes as a reference is arbitrary, so the predictions of the model should not depend on whether $z_{l}$ refers to cations or anions (this is nothing more than a choice of how the crystal is partitioned into unit cells). However, since (110) bonds lie to the left of anions but to the right of cations, switching to a cation reference interchanges the $B$ and $F$ operators in $V_{B F}(z)$. This changes the sign of the Pockels-effect matrix element in Eq. (1) of Ref. [1], a clear indication that the model is physically invalid.

All of these problems can be avoided if one keeps in mind a simple but often overlooked fact: An envelope function is not an independent entity - it is a quantity derived from the microscopic wave function [3].

This work was supported by Hong Kong RGC Grant No. DAG96/97.SC38.

Bradley A. Foreman

Department of Physics

Hong Kong University of Science and Technology

Clear Water Bay, Kowloon

Hong Kong, China

Received 6 July 1998 [S0031-9007(98)08363-X]

PACS numbers: 73.20.Dx, 78.20.Fm, 78.20.Jq, 78.66.-w

[1] O. Krebs and P. Voisin, Phys. Rev. Lett. 77, 1829 (1996).

[2] O. Krebs et al., Phys. Rev. Lett. 80, 5770 (1998).

[3] J. M. Luttinger and W. Kohn, Phys. Rev. 97, 869 (1955).

[4] M. G. Burt, J. Phys. Condens. Matter. 4, 6651 (1992).

[5] B. A. Foreman, Phys. Rev. Lett. 81, 425 (1998).

[6] E. L. Ivchenko, A. Yu. Kaminski, and U. Rössler, Phys. Rev. B 54, 5852 (1996). 